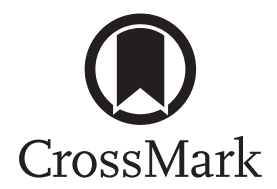

\title{
An informative intragenic microsatellite marker suggests the IL-1 receptor as a genetic modifier in cystic fibrosis
}

\author{
To the Editor:
}

Recent studies in mice with cystic fibrosis (CF)-like lung disease identified interleukin (IL)-1 receptor (IL-1R) signalling as an important pathway triggering neutrophilic airway inflammation that constitutes a key risk factor in the onset and progression of lung disease in patients with CF [1-4]. These studies demonstrated that CF-like airway mucus obstruction causes epithelial hypoxia and necrosis, which in turn leads to the release of IL- $1 \alpha$ from dying cells and activation of IL-1R signalling triggering neutrophilic inflammation and structural lung damage in vivo $[1,5]$. Further, necrotic epithelial cells were detected in mucus-obstructed airways in lung sections from patients with CF [1]. Hypoxic cell death is a well-established trigger of sterile neutrophilic inflammation in many other organs and previous studies have identified IL-1R signalling as a key pathway required for triggering this inflammatory response to dying cells [6]. In addition, the gene encoding the IL-1R ligand IL-1 $\beta$ that is induced by bacterial infection has been identified as a genetic modifier of CF by independent North American and European CF modifier studies $[7,8]$. Collectively, these studies suggest that IL-1R signalling may play an important role in the pathogenesis of neutrophilic inflammation that is invariably detected in the airways of patients with $\mathrm{CF}$, in the absence and presence of bacterial infection $[2,3]$. However, the role of IL-1R and its association with disease severity in patients with CF remains unknown.

The aim of this study was therefore to assess the potential role of the $I L 1 R$ gene, encoding a central molecule in the IL-1R signalling pathway, as a modifier of disease severity in patients with CF. To achieve this goal, we genotyped the informative microsatellite marker IL1RSat located within intron 1 of $I L 1 R$ in several independent CF patient cohorts and a population control derived from the PopGen Biobank (figure 1). We amplified the (AAT)n repeat motif IL1RSat characterised by high polymorphism information content, which is a prerequisite for a marker to capture functional genetic variation at linked sites (figure 1a). We considered IL1RSat to be a neutral genetic marker and used it as a surrogate marker for other variants in its vicinity that are co-inherited together with IL1RSat because of linkage disequilibrium. We genotyped three independent CF patient cohorts at IL1RSat: CF patients of the European Twin and Sibling Study (EUCFTSib) who were born between 1957 and 1990 (mean year of birth 1979) and recruited in 1996 whereby only pairs of two siblings old enough to perform lung function measurements were accepted [8], and patients from the CF centres in Munich $(<1970 \mathrm{M})$ and Hannover $(<1970 \mathrm{H})$ who were born before 1970 and survived at least until 1990. The EUCFTSib population contains 39 patient pairs that were selected from a total of 318 pairs using the following strategy: anthropometry and lung function data were used to rank all individuals according to their disease severity [8]. These selected pairs represent both mild and severe CF disease, whereby the individuals' phenotypes are described by clinical parameters for growth and lung function and all selected patients from EUCFTSib rank below the 25th centile (severe) or above the 75th centile (mild) for both parameters [8]. Pairs were included into the analysis of CF-modifying genes if 1) they were F508del homozygous in order to minimise the effect of the CFTR genotype on phenotype variability, thus emphasising the influence of non-CFTR genes; and 2) if the pair's phenotype was either extremely concordant (concordant mildly affected, 13 pairs; concordant severely affected, 12 pairs) or discordant (14 pairs) [8]. As a higher impact of inherited factors on phenotype manifestation can be expected when studying extreme phenotypes [11, 12], this strategy leads to an enrichment of functional variants in CF-modifying genes in EUCFTSib.

@ERSpublications

IL1R modifies disease severity in CF patients, emphasizing the significance of IL-1 signalling for CF pathogenesis http://ow.ly/pIN730gL10G

Cite this article as: Stanke F, Hector A, Hedtfeld S, et al. An informative intragenic microsatellite marker suggests the IL-1 receptor as a genetic modifier in cystic fibrosis. Eur Respir J 2017; 50: 1700426 [https:// doi.org/10.1183/13993003.00426-2017]. 
a)
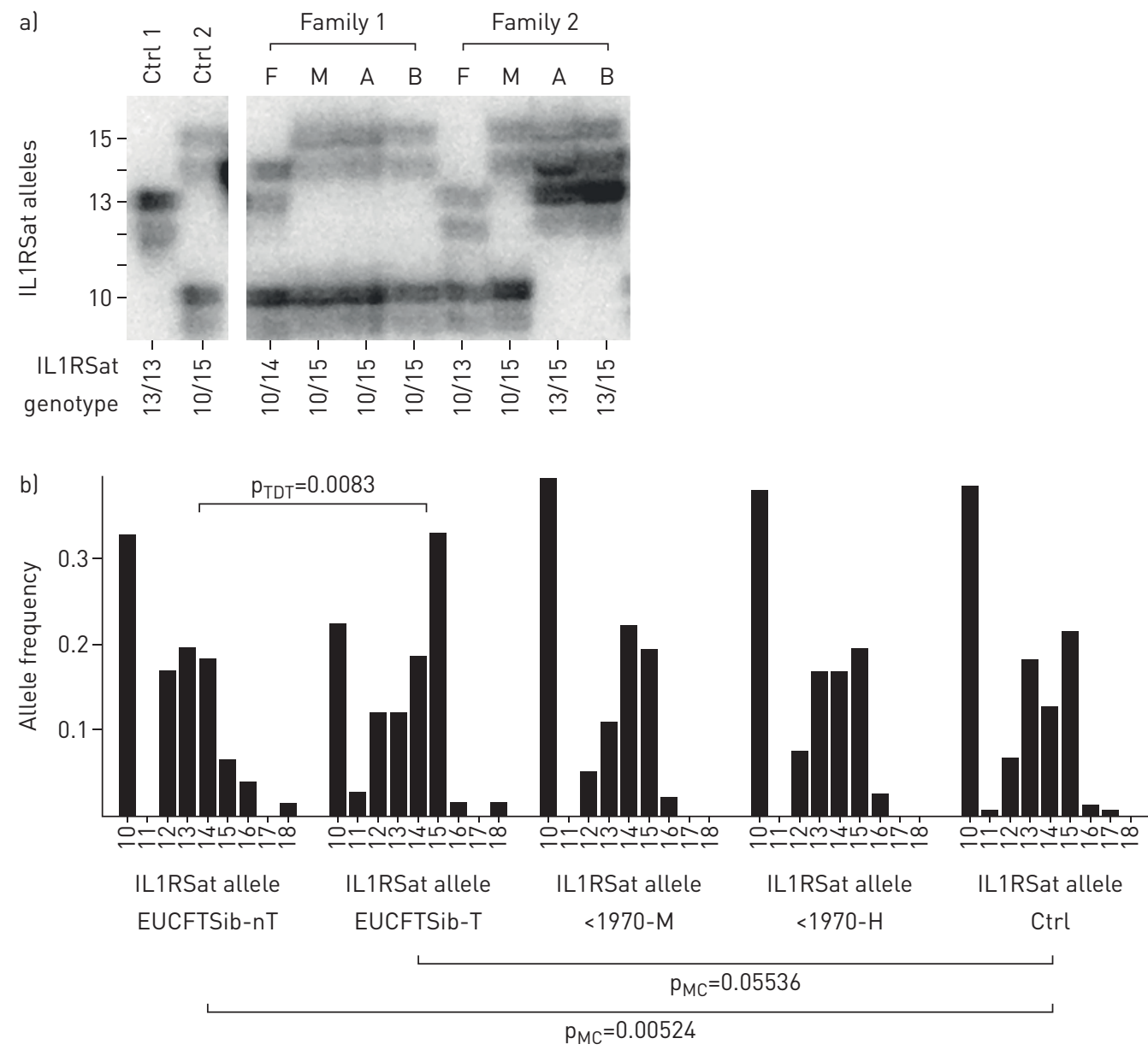

FIGURE 1 An informative microsatellite marker within intron 1 of the interleukin 1 receptor (IL1R) gene is associated with survival in patients with cystic fibrosis. This microsatellite IL1Rsat was selected for genotyping from a set of eight microsatellite motifs, all located within intron 1 of IL1R that were tested on five unrelated control samples. IL1RSat is located within intron 1 of IL1R, starting at nucleotide 102725407 of the chromosome 2 reference sequence GRCh37.p13, with primers [5'-tcactctgactcccagctca-3' and $5^{\prime}$-gcggtacctaaaaccaggtc- $3^{\prime}$ ) surrounding an (AAT)n repeat motif. We observed signals that vary in size by three nucleotides, describing a total of nine IL1RSat alleles, indicating that the number of AAT repeat motifs differs between individuals in a population and is suitable to reflect at least partially the genetic background of the IL1R gene. a) IL1RSat genotyping primary data. Two control samples were used on all analyses to identify the allele by size. Assignment of the IL1RSat genotype to observed signals is shown for two families from the European CF Twin and Sibling (EUCFTSib) study. For example, in family 2, alleles transmitted from parents to offspring are 13 (paternal) and 15 (maternal) while non-transmitted alleles are 10 (paternal) and 10 (maternal). b) IL1RSat allele distributions among cystic fibrosis (CF) families and patients. The interleukin 1 receptor (IL1R) gene was interrogated as a genetic modifier of CF by genotyping of the informative microsatellite marker IL1RSat within intron 1 of IL1R in F508del-CFTR homozygous patients from EUCFTSib (39 families with 78 patients), from the CF centre Munich (<1970-M, 18 unrelated patients) and from the CF centre in Hannover $(<1970-\mathrm{H}, 39$ unrelated patients). Patients of these independent cohorts had survived at least until school age laffected patient pairs of EUCFTSib; mean age at day of recruitment 17 years, range 639 years) or adulthood (unrelated patients born prior to 1970 who have survived until recruitment for DNA analysis in 1990 or later; <1970-M and <1970- $\mathrm{H}$; mean age in 1990, 24 years, range 21-32 years) and a population control from Schleswig-Holstein in Germany (Ctrl, 94 individuals). Distributions of IL1RSat allele frequencies are shown for transmitted (EUCFTSib-T) and non-transmitted (EUCFTSib-nT) alleles in families of the European CF Twin and Sibling Study and for independent patient cohorts followed at the CF centres in Munich $(<1970-M)$ and Hannover $(<1970-H)$. Genetic data of CF patients and their parents in the EUCFTSib were analysed with the software package FAMHAP, which corrects for sibpair dependency in association and linkage studies ( $\mathrm{p}_{\mathrm{TDT}}$ ) [9]. Allele distributions between cohorts of unrelated patients and controls were directly compared using Monte Carlo simulation with CLUMP ( $\left.p_{M C}\right)[10]$.

Patients from the other two CF cohorts were F508del-CFTR homozygous but not selected for any disease manifestation to cover the entire spectrum of disease severity among the survivors. For unrelated patients, epidemiological data on CF birth cohorts provide an estimate on how mortality rates influence the proportion of patients that could be recruited in our study. An analysis of survival of CF patients in the 
UK during the time period 1977 to 1985 reported a 50\% survival at 19 years of age [13]. These UK survivors showed a birth year range similar to our two German cohorts of unrelated patients, indicating that we would have included only $\sim 50 \%$ of the CF population if we had recruited those survivors in $1977-$ 1985 and that even fewer would have been enrolled in 1990 or later. There is no corresponding epidemiologic data available that describe the survival among CF sibling pairs, but the chance to recruit two life siblings simultaneously from a birth cohort that exhibits significant mortality [13, 14] is even lower (and thus the survivor effect higher) than the probability to recruit unrelated patients from the same birth cohort. We therefore expect that the inclusion criterion "two surviving siblings" contributed to a further enrichment of benign alleles in the EUCFTSib population compared with individual patients.

We first used the transmission disequilibrium test (TDT), originally developed by SPIELMAN et al. [15] in their discovery of the diabetes-causing locus, to compare the distribution of IL1RSat allele frequencies between the inherited genetic information transmitted to the CF patients (transmitted alleles) versus the information that was retained in the parental generation (non-transmitted alleles) from CF families of EUCFTSib. We found a transmission disequilibrium with allele 15 at IL1RSat over-represented in the recruited CF siblings $\left(\mathrm{p}_{\mathrm{TDT}}=0.0083\right.$ ), annotating the IL1RSat allele 15 as a benign variant associated with survival of CF patient until enrolment into the study (mean age 17 years, range 6-39 years). The allele distribution in the control population (Ctrl) differed from the IL1R1Sat allele distribution on the transmitted alleles (i.e. surviving $\mathrm{CF}$ patients) $\left(\mathrm{p}_{\mathrm{MC}}=0.055\right)$, as well as the non-transmitted alleles $\left(\mathrm{p}_{\mathrm{MC}}=0.00524\right)$ of the parents from EUCFTSib (figure 1). In independent cohorts of CF patients that were born before 1970 and survived until at least 1990, besides the ubiquitous allele 10 that accounted for a third of the chromosomes in all populations, IL1RSat alleles $14(<1970 \mathrm{M})$ and $15(<1970 \mathrm{H})$ were the most frequently observed alleles (figure 1), albeit this trend towards a similar pattern of enrichment of allele 15 at IL1RSat did not reach statistical significance $\left(\mathrm{p}_{\text {best }}=0.08\right)$ with the number of patients that were available for this study.

The TDT builds on the comparison of transmitted and non-transmitted alleles within nuclear families as an association study with internal controls enabling the identification of disease-causing genes [15]. For the monogenic disease CF that is caused by the CFTR gene, we previously showed that the TDT can also be used to detect the influence of additional genetic modifiers that have an impact on patient survival prior to enrolment in cross-sectional studies [8]. The distortion of allele frequencies because of a cross-sectional recruitment strategy in the CF patient population, for which an improvement in disease management and patient survival could be accomplished within the last decades, has been noticed before through an enrichment of mild pancreatic sufficient-associated CFTR mutations that account for $18 \%$ of CFTR alleles for patients born in 1966 or earlier who were recruited for CFTR mutation analysis in 1990 or later [16]. Moreover, a survivor effect has been detected through an enrichment of TGFB1-Leu10 in a F508del-CFTR homozygous CF patient population $(\mathrm{p}=0.066)$ [16] and by TDT among EUCFTSib at D12S889 in the TNF $\alpha$ receptor gene TNFR1 $(\mathrm{p}=0.0067)$, in the gene encoding the $\gamma$-subunit of the amiloride-sensitive sodium channel SCNN1G $(\mathrm{p}=0.0034)$, and, by default, at the CFTR locus $\left(\mathrm{p}=10^{-6}\right)$ as an indication of the CF disease-causing gene itself [8].

In summary, we have assessed whether a highly polymorphic microsatellite in intron 1 of the ILIR gene shows enrichment of a specific allele in patients with CF who have survived to take part in the study. Our finding that the informative microsatellite marker within intron 1 of $I L 1 R$ detects a survival advantage for patients with $\mathrm{CF}$, revealed through a transmission disequilibrium in patient cohorts (figure 1) with a survivor-dependent recruitment bias [13, 14], supports involvement of IL-1R in CF pathogenesis. This concept is in agreement with an important role of the IL-1R signalling pathway in the pathogenesis of neutrophilic inflammation, as well as findings from previous studies that identified $I L 1 B$ encoding the IL-1R ligand IL-1 $\beta$ as a modifier gene in CF [7, 8]. Earlier, LEvy et al. [7] described an association signal with lung disease at IL1R among female F508del-CFTR homozygous patients from the University of North Carolina and Case Western Reserve (UNC/CWRU) Cohort. Taken together, these studies draw attention to the role of the IL-1R signalling pathway, either activated by IL- $1 \alpha$ released from hypoxic cells in mucus-obstructed airways or by IL- $1 \beta$ induced by bacterial infection $[1,5,6,17]$, in the pathogenesis of chronic neutrophilic inflammation causing progressive lung damage in patients with CF [1-4]. These findings from genetic and functional studies also indicate that inhibition of IL-1R signalling may be a promising anti-inflammatory strategy in CF and potentially other lung diseases associated with chronic airway mucus obstruction and neutrophilic inflammation.

Frauke Stanke $^{1,2}$, Andreas Hector ${ }^{3}$, Silke Hedtfeld ${ }^{1}$, Dominik Hartl ${ }^{3}$, Matthias Griese ${ }^{4,5}$, Burkhard Tümmler ${ }^{1,2}$ and Marcus A. Mall ${ }^{6,7,8}$

${ }^{1}$ Dept of Pediatric Pneumology, Neonatology and Allergology, Hannover Medical School, Hannover, Germany.

${ }^{2}$ Biomedical Research in Endstage and Obstructive Lung Disease Hannover (BREATH), German Centre for Lung Research, Hannover, Germany. ${ }^{3}$ Pediatric Infectiology, Immunology, and Cystic Fibrosis, Children's Hospital of the 
University of Tübingen, Tübingen, Germany. ${ }^{4}$ Dept of Pediatric Pneumology, Hauner Children's Hospital, University of Munich, Munich, Germany. ${ }^{5}$ Comprehensive Pneumology Center (CPC), German Center for Lung Research (DZL), Munich, Germany. ${ }^{6}$ Dept of Translational Pulmonology, Translational Lung Research Center Heidelberg (TLRC), German Center for Lung Research (DZL), University of Heidelberg, Heidelberg, Germany. ${ }^{7}$ Division of Pediatric Pulmonology and Allergy and Cystic Fibrosis Center, Dept of Pediatrics, University of Heidelberg, Heidelberg, Germany. ${ }^{8}$ Dept of Pediatric Pulmonology and Immunology, Charité-Universitätsmedizin Berlin, Berlin, Germany.

Correspondence: Frauke Stanke, Dept of Pediatric Pneumology, Neonatology and Allergology, Hannover Medical School, Carl-Neuberg-Strasse 1, 30625 Hannover, Germany. Email: mekus.frauke@mh-hannover.de

Received: Feb 272017 | Accepted after revision: Oct 072017

Support statement: This study was supported by grants from the German Federal Ministry of Education and Research (82DZL00401, 82DZL004A1, 82DZL00201 and 82DZL002A1). Funding information for this article has been deposited with the Crossref Funder Registry.

Conflict of interest: Disclosures can be found alongside this article at erj.ersjournals.com

Acknowledgements: We thank the organisers of the PopGen Biobank (https://www.epidemiologie.uni-kiel.de/ biobanking/biobank-popgen) for providing DNA samples from a control population.

Author contributions: conception and design of the study: F. Stanke, B. Tümmler, M.A. Mall; acquisition, analysis and interpretation of data: F. Stanke, A. Hector, S. Hedtfeld, D. Hartl, M. Griese, B. Tümmler, M.A. Mall; drafting the article or revising it critically for important intellectual content: F. Stanke, M.A. Mall.

\section{References}

1 Fritzsching B, Zhou-Suckow Z, Trojanek JB, et al. Hypoxic epithelial necrosis triggers neutrophilic inflammation via IL-1 receptor signaling in cystic fibrosis lung disease. Am J Respir Crit Care Med 2015; 191: 902-913.

2 Sly PD, Gangell CL, Chen L, et al. Risk factors for bronchiectasis in children with cystic fibrosis. $N$ Engl J Med 2013; 368: 1963-1970.

3 Sagel SD, Wagner BD, Anthony MM, et al. Sputum biomarkers of inflammation and lung function decline in children with cystic fibrosis. Am J Respir Crit Care Med 2012; 186: 857-865.

4 Montgomery ST, Mall MA, Kicic A, et al. Hypoxia and sterile inflammation in cystic fibrosis airways: mechanisms and potential therapies. Eur Respir J 2017; 49: 1600903.

5 Mall MA, Harkema JR, Trojanek JB, et al. Development of chronic bronchitis and emphysema in $\beta$-epithelial $\mathrm{Na}^{+}$ channel-overexpressing mice. Am J Respir Crit Care Med 2008; 177: 730-742.

6 Chen CJ, Kono H, Golenbock D, et al. Identification of a key pathway required for the sterile inflammatory response triggered by dying cells. Nat Med 2007; 13: 851-856.

7 Levy H, Murphy A, Zou F, et al. IL1B polymorphisms modulate cystic fibrosis lung disease. Pediatr Pulmonol 2009; 44: 580-593.

8 Stanke F, Becker T, Kumar V, et al. Genes that determine immunology and inflammation modify the basic defect of impaired ion conductance in cystic fibrosis epithelia. J Med Genet 2011; 48: 24-31.

9 Herold C, Becker T. Genetic association analysis with FAMHAP: a major program update. Bioinformatics 2009; 25: $134-136$

10 Sham PC, Curtis D. Monte Carlo tests for associations between disease and alleles at highly polymorphic loci. Ann Hum Genet 1995; 59: 97-105.

11 Risch N, Zhang H. Extreme discordant sib pairs for mapping quantitative trait loci in humans. Science 1995; 268: $1584-1589$.

12 Dolan CV, Boomsma DI. Optimal selection of sib pairs from random samples for linkage analysis of a QTL using the EDAC test. Behav Genet 1998; 28: 197-206.

13 British Paediatric Association Working Party on Cystic Fibrosis. Cystic fibrosis in the United Kingdom 1977-85: an improving picture. BMJ 1988; 297: 1599-1602.

14 Elborn JS, Shale DJ, Britton JR. Cystic fibrosis: current survival and population estimates to the year 2000. Thorax 1991; 46: 881-885.

15 Spielman RS, McGinnis RE, Ewens WJ. Transmission test for linkage disequilibrium: the insulin gene region and insulin-dependent diabetes mellitus (IDDM). Am J Hum Genet 1993; 52: 506-516.

16 Becker T, Jansen S, Tamm S, et al. Transmission ratio distortion and maternal effects confound the analysis of modulators of cystic fibrosis disease severity on 19q13. Eur J Hum Genet 2007; 15: 774-778.

17 Dinarello CA, Simon A, van der Meer JW. Treating inflammation by blocking interleukin-1 in a broad spectrum of diseases. Nat Rev Drug Discov 2012; 11: 633-652. 